\title{
Higgs Boson Discovery Versus Sparticles Prediction: Impact on the pMSSM's Posterior Samples from a Bayesian Global Fit
}

\author{
Shehu S. AbdusSalam ${ }^{1, *}$, Debajyoti Choudhury ${ }^{2}$ \\ ${ }^{1}$ The Abdus Salam International Centre for Theoretical Physics, Strada Costiera 11, Trieste 34014, Italy \\ ${ }^{2}$ Department of Physics and Astrophysics, University of Delhi, Delhi 110007, India \\ *Corresponding Author: shehu@ictp.it
}

Copyright (C2014 Horizon Research Publishing All rights reserved.

\begin{abstract}
The signal strength of the recently discovered Higgs boson-like particle in the diphoton channel seemingly constrains physics beyond the standard model to a severe degree. However, the reported signal strength is prone to possible underestimation of uncertainties. We propose a discriminant that is relatively free of many of the theoretical uncertainties, and use this to gauge the impact on the phenomenological MSSM. A Bayesian global fit to all the pre-LHC data results in posterior distributions for the masses that are neither very restrictive, nor sufficiently prior-independent (except for the Higgs and stop masses). The imposition of the Higgs data, on the other hand, yields interesting and nearly prior-independent constraints. In particular, the existence of some light superpartners is favoured.
\end{abstract}

Keywords sparticle, Bayesian, minimal supersymmetric standard model, Higgs particle: mass

\section{Introduction}

The recent discovery of a $126.5 \mathrm{GeV}$ or $125.0 \mathrm{GeV}$ Higgs boson-like state, respectively by the ATLAS and CMS collaborations [1,2], at the LHC marks the beginning of an exciting scientific era for understanding the mechanism responsible for electroweak symmetry breaking and for endeavour in search for new, beyond the standard model (BSM), physics. For a standard model-like Higgs boson, the search strategies at the LHC involve, in order of decreasing sensitivity, production processes driven by gluon fusion $g g \rightarrow H$, vector boson fusion $q \bar{q} \rightarrow H q q$, associated production with a vector boson $q \bar{q} \rightarrow V H$ and the associated production with a top-quark pair $g g \rightarrow t \bar{t} H$. Further to production, the main search channels are defined by decay modes into $\gamma \gamma, Z Z^{*}, W W^{*}, b \bar{b}$ and $\tau^{+} \tau^{-}$. Subsequent decays of the $W$ - and $Z$-bosons with at least one leptonic decay are also considered. For the experiments with the Tevatron collider, the main production modes, for the most sensitive search topologies, are the associated production of a Higgs with a vector boson $q \bar{q} \rightarrow V H$, with the subsequent decay of the Higgs into a $b \bar{b}$ pair, and the $g g$-fusion production with the subsequent decay into two $\mathrm{W}$ bosons which then decay leptonically. The LHC discovery has supporting evidence from the Tevatron experiments (see e.g. [3] and references therein) where the excess seen is in accord with a 115 to $135 \mathrm{GeV}$ Higgs boson.

Usually, the Higgs boson signal is quantified by the production cross section times the relevant decay-channel branching fraction to give the signal strength,

$$
\mu_{X}=\frac{\sigma(g g \rightarrow h) B r(h \rightarrow X)}{\sigma(g g \rightarrow h)_{S M} B r(h \rightarrow X)_{S M}} .
$$

Here $X=\gamma \gamma, Z Z^{*}, W W^{*}, b \bar{b}$, or $\tau^{+} \tau^{-}$. Of the Higgs boson decay channels reported by the experimental collaborations, $\mu_{\gamma \gamma}$ apparently makes for a very strong BSM-constraining observable. However, as argued in Ref. [4], it is relevant to question whether the apparent excess is actually due to new physics effect or due to under-estimations in theoretical uncertainties. The question would also propagate directly to any analysis that employs the reported signal strength. In order to avoid this and for the purpose of allowing robust conclusions to be derived from the discovery data, we are going to propose a discriminant between new physics and the standard model (SM), which should be largely free of the mentioned source of uncertainties. 
We consider, here, the R-parity conserving minimal supersymmetric standard model (MSSM) as a template for BSM. Rather than appeal to a particular scenario for supersymmetry breaking and/or make an Ansatz for the parameters at some high scale, we, instead, use the phenomenological MSSM (pMSSM) parametrisation [5,6] to characterise the supersymmetric sector. Although this is almost the most general scenario given the filed content, it is, nonetheless, subject to very many constraints. For example, Refs. $[7,8]$ had used all pre-LHC data to arrive at Bayesian posterior distributions for the superpartner masses. We discuss here how the mass of the Higgs resonance and the information on the signal strengths (in particular, the robust discriminant) can be used to further refine the posterior to yield some very interesting clues about the pMSSM spectrum. Other discussions concerning LHC constraints on the pMSSM were effected in Refs. [9-13].

Several studies have been made of the MSSM Higgs sector in light of the observed excess (Refs. [13-19] comprise a non exhaustive list). in the diphoton channel signal strength or the effect of light sparticles in altering it. Some of these attempts are based on random scans in the pMSSM parameter space while others restrict themselves to sub-spaces or hyper-planes of the MSSM parameters space. In this article we make use of a complete set of pMSSM points that came out of statistically convergent Bayesian fits. The aim is to derive the implications of the recent LHC discovery on the pMSSM posterior distributions by gauging its effect on the sparticle masses. In the the rest of this section we introduce the BSM discriminant for the analysis. In Section 2 we give a brief recapitulation of the pMSSM's posterior distributions for sparticles masses, Higgs boson signal strengths, and the proposed discriminant predictions from a pre-LHC Bayesian fit to data. Section 3 describes the implementations and implications of the Higgs boson data on the pMSSM predictions.

\subsection{A useful discriminant}

The ratios $\mu_{X}$, see eq.(1), represent good measures for possible deviations from the SM and have, indeed, been exploited to discriminate against BSM models. However, such an exercise ought to be approached with care, for it is yet early days of Higgs physics and the statistics are still low. A naive adoption of the current bounds would indicate a significant tension with the SM itself [20]. Of particular concern are the theoretical errors pertaining to the choice of the parton distributions and the renormalization $\left(Q_{R}\right)$ and factorization $\left(Q_{F}\right)$ scales. These uncertainties can be large and have been addressed in Ref. [21]. The LHC Higgs cross section working group recommends [22] that in convoluting the errors, these should be added linearly. However, both ATLAS and CMS have added them in quadrature. As Ref. [4] explicitly demonstrates, adopting the more conservative approach of linear addition would substantially increase the error bars to the extent of a $1 \sigma$ agreement with the SM. A further uncertainty pertains to the use of an effective field theory (in the limit of an infinitely heavy top) used for many of the higher-order calculations.

This criticism brings into question the naive use of $\mu_{X}$. However, note that the uncertainty applies primarily to the production process under consideration viz. $p p \rightarrow h$ (driven, at the lowest order, through gluon-fusion). In fact, as far as non-hadronic final states (diphoton, and four-lepton, charged or neutral) are concerned, this is an exact statement as quantum chromodynamics (QCD) corrections can occur exclusively in the initial state alone. For $p p \rightarrow h \rightarrow b \bar{b}$, though, one could have gluon exchanges between the initial and final state particles, thereby engendering non-universal dependence on the parton density. Nevertheless, as is well-known, such diagrams result in only a subdominant component of the higher-order QCD corrections, and, thus, even for the $p p \rightarrow h \rightarrow b \bar{b}$ case, the dependence is identical to a very good approximation. The same argument holds for $p p \rightarrow h \rightarrow V V^{*}$ with the gauge boson(s) going into jets.

In other words, if we consider only those final states that are primarily driven by Higgs-production through gluon-gluon fusion, the ratios of ratios $^{1}$, namely,

$$
R_{i j} \equiv \frac{\mu_{i}}{\mu_{j}}
$$

are essentially free of the aforementioned uncertainties due to the choice of $Q_{R, F}$ as well as the choice of parton distributions $^{2}$. Here $i$ and $j$ represent distinct choice from $X=\gamma \gamma, Z Z^{*}, W W^{*}, b \bar{b}$, or $\tau^{+} \tau^{-}$. The same argument would hold for other systematic errors that are factorisable, or are nearly universal. A prime example is afforded by the uncertainty in the luminosity measurement.

Thus, it makes eminent sense to consider all the independent ratios $R_{i j}$, and, at best, one individual $\mu_{i}$, for, together, they embody the same information as the set $\left\{\mu_{i}\right\}$. There is, of course, a price to pay: the relative statistical errors in $R_{i j}$ would be larger than those in the individual $\mu_{i}$. Furthermore, certain statistical errors in $\mu_{i}$ are correlated ${ }^{3}$, and, unless the correlation matrix is available, a naive combination would tend to overestimate the error in $R_{i j}$. This, certainly, is the case in the current context in the absence of sufficient public information about the ATLAS and CMS analyses.

\footnotetext{
${ }^{1}$ Ref. [23] too had raised the possibility of using such ratios, without putting it into practice. Refs [24-26] also used similar ratios for suppressing systematic errors.

${ }^{2}$ It is also worthwhile to note that even the ratios of, say, gluon-fusion and vector-boson fusion cross-sections also profit on this account, though not to the same high degree as is applicable here.

${ }^{3} \mathrm{An}$ example would be those pertaining to the electromagnetic calorimeter measurements, relevant to both the diphoton channel and the $V V^{*}$ modes containing electrons.
} 
Table 1. The ATLAS [1] and CMS [2] magnitudes for the Higgs boson production signal strengths.

\begin{tabular}{|c|l|l|}
\hline \hline Search channel & ATLAS $\mu$ at $m_{h}=126.5 \mathrm{GeV}$ & CMS $\mu$ at $m_{h}=125.0 \mathrm{GeV}$ \\
\hline$H \rightarrow \gamma \gamma$ & $1.8 \pm 0.5,4.5 \sigma$ & $1.6 \pm 0.4,4.6 \sigma$ \\
$H \rightarrow Z Z^{*}$ & $1.2 \pm 0.6,3.6 \sigma$ & $0.7_{-0.3}^{+0.4}, 3.2 \sigma$ \\
\hline \hline
\end{tabular}

Nonetheless, for our analyses we shall consider such ratios $R_{i j}$. Of particular interest here is the ratio of the two reported discovery channels, namely four-lepton and diphoton. The ATLAS and CMS ratios for the same, as shown in Table 1 , are consistent with each other within about $0.8 \sigma$, and combining the two, we have,

$$
R_{Z Z ; \gamma \gamma} \equiv \frac{\mu_{Z Z}}{\mu_{\gamma \gamma}}=0.56 \pm 0.25
$$

which, in itself, is consistent with the $\mathrm{SM}$ within $2 \sigma$. Yet, the very fact that the central value of $R_{Z Z ;} \gamma \gamma$ is substantially below the SM expectation would turn out to have rather interesting consequences. It should be realized that this deviation is primarily driven by the fact that $\mu_{\gamma \gamma}$ is significantly larger than unity; yet, unlike the latter, this observable is largely free from the aforementioned theoretical errors. Moreover, the error in eq.(3) has been somewhat overestimated and would be reduced substantially once the error correlation matrix is available. This renders any inference drawn from the use of eq.(3) to be conservative in nature.

Similarly, we also have

$$
R_{Z Z ; W W} \equiv \frac{\mu_{Z Z}}{\mu_{W W}}=0.951_{-0.463}^{+0.531}
$$

which is rather consistent with the SM. And while $p p \rightarrow H \rightarrow \tau^{+} \tau^{-}$seems to be much smaller than the SM expectations, the current error bars are too large for the observable to be of any consequence.

\section{A brief recapitulation of the pMSSM and its predictions}

Within the MSSM, the masses and interactions of the Higgs bosons are strongly affected by loop corrections, in particular from the sector of the third-generation quarks/squarks. Here, we consider the predictions for the Higgs bosons in the frame of the phenomenological MSSM (pMSSM) where a non biased approach to the MSSM parametrisation is adopted and with the parameters defined and simultaneously varied at the electroweak scale, the energy scale which the LHC is now probing. For the pMSSM fit, the parametrisation is completely decoupled from the details of the physics responsible for the breaking of SUSY. Only real soft SUSY breaking terms were considered, with all off-diagonal elements in the sfermion mass terms and trilinear couplings set to zero, and the first-and second-generation soft terms equalised ${ }^{4}$, leading to a set of 20 parameters:

$$
M_{1,2,3} ; m_{\tilde{f}_{Q, U, D, L, E}}^{3 r d g e n}, m_{\tilde{f}_{Q, U, D, L, E}}^{1 s t / 2 n d g e n} ; A_{t, b, \tau, \mu=e}, m_{H_{u, d}}^{2}, \tan \beta,
$$

where $M_{1}, M_{2}$ and $M_{3}$ are the gaugino mass parameters; $m_{\tilde{f}}$ are the sfermion mass parameters. $A_{t, b, \tau, \mu=e}$ represent the trilinear scalar couplings while the Higgs-sector parameters are specified by $m_{H_{1}}^{2}, m_{H_{2}}^{2}$, tan $\beta$ and sign $(\mu)$. The set of observables used for the pre-LHC global fits [7,8] are the CDM relic density, electroweak physics observables (EWPOs) and B-physics observables:

$$
\begin{aligned}
\underline{O}= & \left\{m_{W}, \sin ^{2} \theta_{e f f}^{l e p}, \Gamma_{Z}, \delta a_{\mu}, R_{l}^{0}, A_{f b}^{0, l}, A^{l}=A^{e}, R_{b, c}^{0}, A_{f b}^{b, c}, A^{b, c},\right. \\
& B R\left(B \rightarrow X_{s} \gamma\right), B R\left(B_{s} \rightarrow \mu^{+} \mu^{-}\right), \Delta_{0-}, R_{B R\left(B_{u} \rightarrow \tau \nu\right)}, R_{\Delta M_{B_{s}}}, \\
& \left.\Omega_{C D M} h^{2}\right\} .
\end{aligned}
$$

In the absence of direct data, the pMSSM parameter space is weakly constrained in the sense that only a few of the posterior distributions (in particular, those that are strongly linked to the Higgs sector) are approximately prior-independent. The Bayesian fits to data, nonetheless, have an important virtue of indicating how effective a particular data is in constraining a given model in an unambiguous manner. For example, if the LHC data rules out a prior-independent pMSSM prediction, then a large class of MSSM possibilities would have been ruled out at a go instead of trying many little constructed sub-MSSMs. This is what we set to explore in our analysis: (i) check if the Higgs boson discovery data rules out the approximately prior-independent pMSSM predictions in the Higgs sector and (ii) search for any prior-independent impact of the data on the pre-LHC posterior samples.

Unlike the case for sparticle spectrum, the Higgs sector of the pMSSM is strongly constrained by the LEP lower limit and the MSSM's theoretical upper bound on the Higgs mass. This is reflected in the results for the pre-LHC Bayesian fits [7,8] where the Higgs sector results were found to be approximately prior-independent. The light CP-even Higgs boson mass was predicted to be between $117 \mathrm{GeV}$ to $129 \mathrm{GeV}$ at $95 \%$ Bayesian confidence level for both logarithmic and flat priors (i.e., approximately prior independent). This is consistent with the recent

\footnotetext{
${ }^{4}$ This set of assumptions are obviously designed to conform to the very strict constraints on CP violation as well as flavour changing neutral current processes, whether in meson mixings or in rare decays.
} 
observation of a new state compatible with the Higgs boson hypothesis near $125 \mathrm{GeV}$. The constraint on the Higgs sector, in turn, puts a severe constraint on the mass of the scalar top-quark which plays a crucial role in the radiative contribution to the Higgs boson mass. As a result, the statistically convergent Bayesian fits to indirect collider and cold dark matter relic density data for the pMSSM $[7,8]$ reveal that the scalar top-quarks masses have an approximately prior-independent posterior distribution centred around $2 \mathrm{TeV}$.

We assume that the peak discovered by the ATLAS and CMS collaborations is explained by the pMSSM's light $C P$-even Higgs boson. We then compute the signal strengths for the production of the Higgs boson at ${ }^{5} 8 \mathrm{TeV}$ LHC. These are used to check the compatibility and implications of the discovery data on the pMSSM's both approximately prior-independent (such as for the scalar top-quarks) and prior-dependent (as is the case for other sparticle masses) posterior predictions.

\subsection{The pre-LHC predictions for $\mu_{X}$ and $R_{Z Z ; \gamma \gamma}$}

For each pMSSM model, the Higgs boson production cross section $\sigma(g g \rightarrow h)$ and their branching fractions $\operatorname{Br}(h \rightarrow X)$ are computed using the high energy physics package FeynHiggs [27]. From these, the signal strengths $\mu_{X}$ eq.(1) are calculated. The pMSSM posteriors for the signal strengths are shown in Fig. 1. The signal strengths $\mu_{X}$ have a spread encompassing unity, but typically have somewhat reduced values. In particular, the signal strengths for $g g \rightarrow h \rightarrow b \bar{b} / \tau^{+} \tau^{-}$both, largely, tend to be below those expected for the standard model. Naively, this might seem unexpected in view of the fact that the Yukawa coupling in the MSSM is often larger than in the SM (at least for large $\tan \beta$ ). However, this has to be convoluted with the fact that the $g g \rightarrow h$ amplitude is suppressed on account of the cancellation between the top and the stop loops. While this cancellation is less effective for super-heavy stops, such solutions are, typically, less favoured once constraints such as $\Omega_{C D M} h^{2}, B R\left(B \rightarrow X_{s} \gamma\right)$ and $\delta a_{\mu}$ are taken into account. Indeed, this could be gleaned from the pre-LHC pMSSM posteriors as well. The reduction is more pronounced for the log prior fit's posterior distribution since the sparticle masses are lighter. Note that $p p \rightarrow h \rightarrow b \bar{b}$ would, perhaps, never be seen over the large QCD background, while both ATLAS and CMS see fewer candidates for $p p \rightarrow h \rightarrow \tau^{+} \tau^{-}$than would be expected within the SM. Thus, no conclusion can be drawn, as yet, from these two modes.

The $Z Z$ mode strength can be quite consistent with the SM value, with relatively fewer points leading to a suppression. On the other hand, the observed rate too is marginally smaller than that in SM with an error bar sufficiently large to preclude any significant constraint ${ }^{6}$. The situation with the diphoton rate is more dramatic. While, on the face of it, even $\mu_{\gamma \gamma}$ can be consistent with unity, note that this rate largely lies well below the SM one. Part of this can be attributed to the aforementioned suppression in the production cross section. An additional suppression accrues due to the cancellations between the $W$-loop (dominant contribution within the $\mathrm{SM}$ ) and the chargino loops (care must be taken to include the charged-Higgs loops as well) on one hand and the top- and stop-loops on the other. More importantly, the observed rate is significantly above the SM expectation, a situation that is rare within the MSSM. Thus, if we use $\mu_{\gamma \gamma}$ as a constraint, very few points would survive in the pMSSM scan, a situation that is corroborated by Table 2, where we have summarised the relative number of the model points that survive the various experimental observations, individually and in combination.

\section{Higgs discovery impact on pMSSM sparticle predictions}

The Higgs discovery data may be considered to consist of two forms of observables, namely the Higgs boson mass and the signal strengths. Although the pre-LHC data predict $m_{h} \in[117,129] \mathrm{GeV}$ at $95 \%$ C.L. in correspondence with large stop masses as every additional increment in $m_{h}$ beyond $m_{h}=m_{Z}$ (with only the MSSM particle content) needs progressively larger stop masses ${ }^{7}$. This immediately implies that requiring $m_{h}=125-126.5 \mathrm{GeV}$ would impose significant further constraints on the pre-LHC pMSSM posterio distribution, see Table 2. However, given the about $3 \mathrm{GeV}$ errors from theoretical computations [28] one could, for the purpose of the present analysis, consider the Higgs boson mass range of

$$
m_{h}=122-128 \mathrm{GeV}
$$

to be plausible and allowed for the pMSSM predictions.

The experimental values for the Higgs boson signal strengths we use are shown in Table 1. While the excesses do depend on the exact mass bin, it is, nonetheless, useful to combine the two data from the two experiments to yield

$$
1.2 \leq \mu_{\gamma \gamma} \leq 2.3, \quad 0.4 \leq \mu_{Z Z} \leq 1.8
$$

While this (along with eq.(7)) does represent a slight simplification, this is the best that can be done until the two experiments combine their data. After applying various constraints including the new discovery mode signal

\footnotetext{
${ }^{5}$ Although the discovery claims of both ATLAS and CMS are based on a combination of $7 \mathrm{TeV}$ and 8 TeV data, the latter has a somewhat weight on account of both higher cross sections as well as a larger integrated luminosity. Furthermore, the difference between the two runs accounts for little in our analysis.

${ }^{6}$ It should be noted here that the situation with $h \rightarrow W W$ is similar, albeit with even larger error bars.

${ }^{7}$ There is an exception for the "Natural SUSY" scenario which allows for light stops and at the same time satisfying $m_{h} \sim 125$ GeV. This is possible within the pMSSM but is beyond our purpose here.
} 

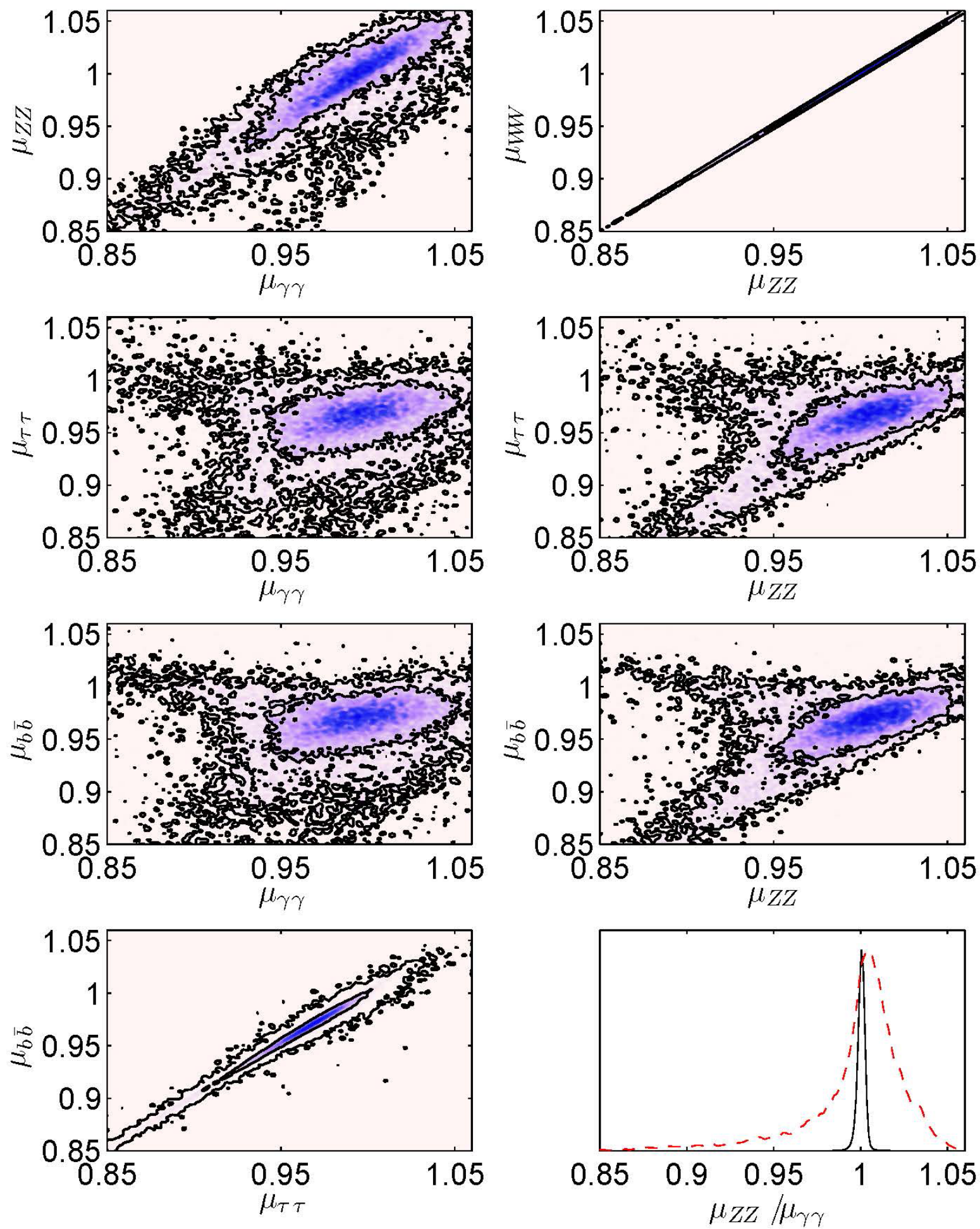

Figure 1. 2-D marginalised posterior distributions for the log prior pMSSM's Higgs boson signal strengths at the LHC with $8 \mathrm{TeV}$ collision energy. The colour spread from light pink to blue represents relative probability spread from zero to unity. The inner and outer contours represent $68 \%$ and $95 \%$ probability regions respectively. The distributions for the flat prior sample is approximately the same but with narrower peaks around unity. The $\mu_{Z Z} / \mu_{\gamma \gamma}$ 1-D marginalised posterior distribution are for the log (red/broken curve) and flat (black/solid curve) prior pMSSM fits.

strengths, the relative amount of the model points that survive various combinations of the experimental cuts eq.(3) and eq.(7) are summarised in Table 2.

As we have already pointed out, the naive imposition of $\mu_{\gamma \gamma}$ (or, for that matter, any of the $\mu_{X}$ ) as a constraint is fraught with danger in view of the uncertainties in the production cross section. On the other hand, the use of $R_{Z Z ; \gamma \gamma}$ (see eq.(3)) largely eliminates these uncertainties, and given its sizable deviation from unity, is expected to lead to more robust claims. As Table 2 shows, the latter constraint is not significantly weaker than the former but, of course, it is far less constraining than the simultaneous imposition of both $\mu_{\gamma \gamma}$ and $\mu_{Z Z}$. 
Table 2. Summary of the relative number of surviving posterior points, from the pre-LHC Bayesian global fits of the pMSSM, after imposing the Higgs discovery data.

\begin{tabular}{|c|l|l|l|}
\hline No. & Constraint & Log prior survive & Flat prior survive \\
\hline 1. & $m_{h}=122-128 \mathrm{GeV}$ & $36.08 \%$ & $41.24 \%$ \\
2. & $m_{h}=125-126.5 \mathrm{GeV}$ & $9.17 \%$ & $9.93 \%$ \\
3. & $R_{Z Z ; \gamma \gamma}=0.56 \pm 0.25$ & $0.30 \%$ & $0.00 \%(3$ points $)$ \\
4. & $2-\sigma R_{Z Z ; \gamma \gamma}$ & $99.90 \%$ & $100.00 \%$ \\
5. & $1.67-\sigma R_{Z Z ; \gamma \gamma}$ & $13.30 \%$ & $0.40 \%$ \\
6. & $m_{h}(1) \& 1.67-\sigma R_{Z Z ; \gamma \gamma}$ & $4.67 \%$ & $0.20 \%$ \\
7. & $1.2 \leq \mu_{\gamma \gamma} \leq 2.0$ & $0.20 \%$ & $0.01 \%(5$ points $)$ \\
8. & $0.4 \leq \mu_{Z Z} \leq 1.8$ & $99.63 \%$ & $99.96 \%$ \\
9. & $m_{h}(1) \& 1-\sigma R_{Z Z ; \gamma \gamma}$ & $0.07 \%$ & $0.00 \%(0$ points $)$ \\
10. & $\left.m_{h}(2) \& 1-\sigma R_{Z Z ; \gamma \gamma}\right)$ & $0.00 \%(2$ points $)$ & $0.00 \%(0$ points $)$ \\
11. & $\mu_{\gamma \gamma} \& \mu_{Z Z}$ & $0.00 \%(2$ points $)$ & $0.01 \%(5$ points $)$ \\
\hline
\end{tabular}

\subsection{Implications}

The effects of the Higgs data on the pMSSM sparticle posterior distributions are shown in Fig. 2 which shows the allowed regions of sparticle masses for the model points that survive constraint no. 6 in Table 2. Note that the distributions for the surviving points are not meant to represent the possible outcome of a full-fledged parameter fit with the Higgs data, since this would require more statistic and convergence. Observations about the effect of the different constraints on the sparticle masses are as follows.

- Only $m_{h}=122-128 \mathrm{GeV}$ as a constraint: On imposing only this, about $40 \%$ of the posterior samples survive and the constraint does not affect the shape of the SUSY-breaking parameters and sparticles posterior distributions. The only exception is for the strong preference for multi-TeV trilinear coupling, $\left|A_{t}\right| \sim 5 \mathrm{TeV}$. The approximate conservation of the posterior distributions reflects the consistency of the pre-LHC fits with the Higgs boson mass.

- Only $R_{Z Z ; \gamma \gamma}$ as a constraint: Although this discriminant shows an experimental central value very different from unity, note that it has yet a large error bar. Consequently, if we only demand a $2 \sigma$ agreement with it, then essentially all the points survive for both $\log$ and flat prior samples. This is so because the pMSSM predictions are centred around unity, which is marginally consistent at $2 \sigma$. The situation changes dramatically, if we strengthen the demand to a $1.67 \sigma$ (corresponding to a $90 \%$ C.L. for the 1-D marginalised distribution) agreement. Now, only about $13.3 \%$ of the $\log$ prior and $0.4 \%$ of the flat prior posterior samples survive. Consequently, we skip the flat prior samples and continue the analysis with only the log prior. The surviving model points show a slight preference for lighter staus, stau-neutrino and 1st/2nd generation squarks, and for slightly heavier $\tilde{\chi}_{4}^{0}, \tilde{\chi}_{2}^{ \pm}$, stops and $\tilde{b}_{2}$. All other sparticle distributions remain approximately unchanged.

- $m_{h}=122-128 \mathrm{GeV}$ as well as $R_{Z Z ; \gamma \gamma}$ at 90\%C.L.: $5.3 \%$ of the log-prior and $0.2 \%$ of the flat-prior posterior samples survive. The sparticles' posterior distributions for the initial log-prior sample set and for the surviving points are shown in Fig.2. As can be seen from the plots, there is a shift in probability towards heavier neutralinos (except for the LSP), charginos and stops. On the other hand, the data show preference for rather lighter 1st/2nd generation squarks. The signal strengths' posterior distributions for the surviving points are shown in Fig. 3.

- Only $R_{Z Z ; \gamma \gamma}$ at $1 \sigma$ : While a demand that a particular observable agree at $1 \sigma$, statistically speaking, makes little sense, we, nonetheless examine this for curiosity. Partly, this exercise is motivated by the realization that, the error in, eq.(3), has most probably been over-estimated, and is likely to go down significantly once the common errors have been factored out. Furthermore, with more data (already on tapes now), the statistical component would reduce substantially. However, working with the currently available data, imposing this implies that only $0.3 \%$ of the log prior posterior samples survive while all of the flat prior samples get ruled out. The surviving points show preference for light (one to few hundred GeVl) staus and stau-neutrinos; preference for heavier $\tilde{t}_{2}$, and moderate $\tan \beta(\lesssim 40)$; and preference for heavy $(\geq 500 \mathrm{GeV}) \tilde{\chi}_{3}^{0}$ and $(\geq 1000 \mathrm{GeV}) \tilde{\chi}_{4}^{0}$ and $\tilde{\chi}_{2}^{ \pm}$. The $m_{\tilde{t}_{1,2}}$ posterior distribution remain approximately unchanged, mainly peaked around $2 \mathrm{TeV}$. With $m_{h}=122-128 \mathrm{GeV}$ and $R_{Z Z ; \gamma \gamma}$ at 1- $\sigma$, the few surviving points have the characteristics of: (i) having $\tilde{\chi}_{1}^{0} \lesssim m_{h} / 2$, (ii) having light $(\lesssim 500 \mathrm{GeV})$ quasi-degenerate light 1st/2nd-generation squarks with the LSP, and (iii) light stau states.

\section{Summary and conclusions}

The signal strengths for the recently discovered Higgs boson-like state are used to propose a BSM discriminant, the ratio of diphoton- to ZZ- channel signal strengths. This has the advantage that sources of theoretical uncertainties cancel almost completely unlike the case for the individual signal strengths. The discriminant is applied to 

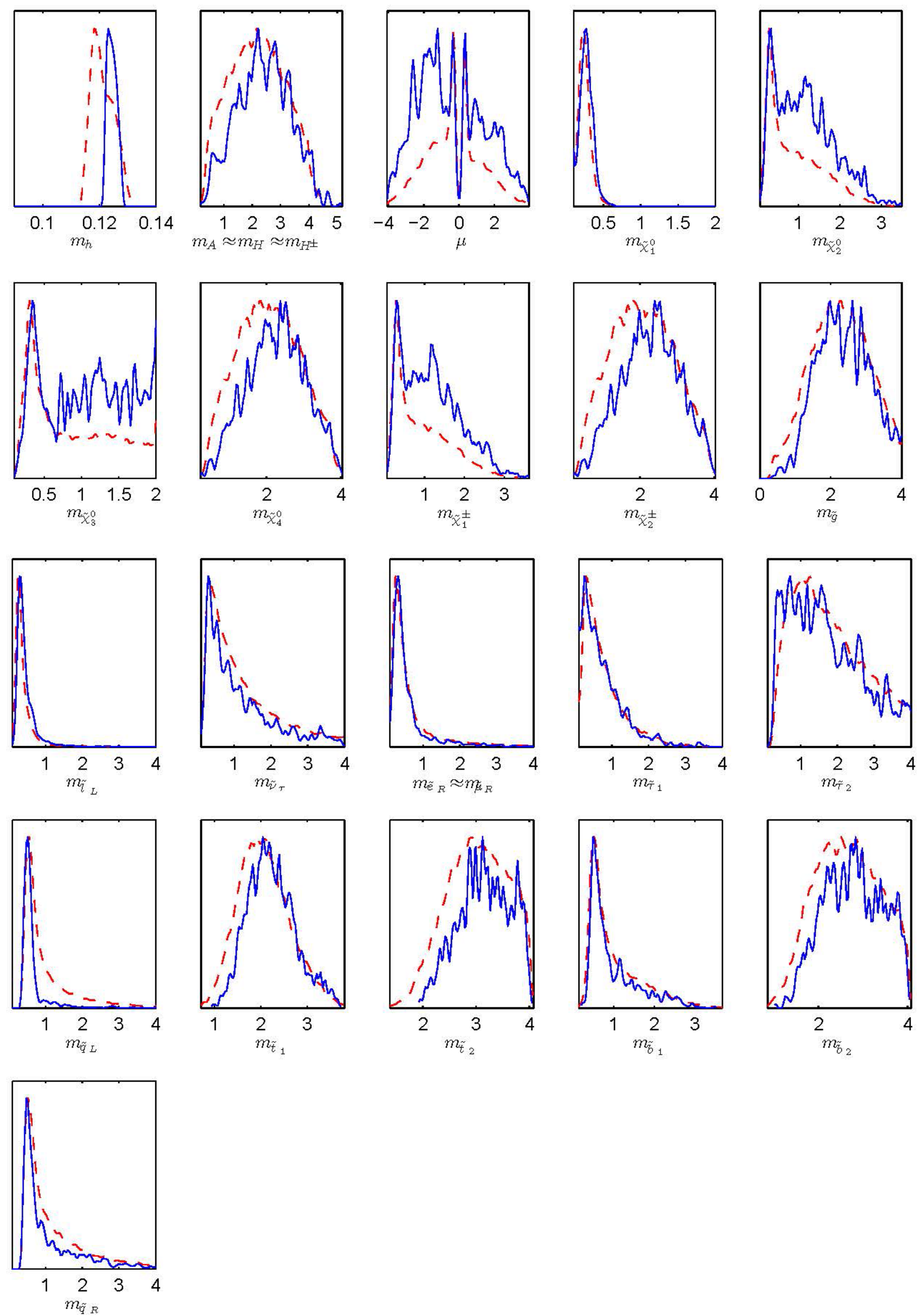

Figure 2. The plots compare the log prior pMSSM sparticle masses' marginalised 1-dimensional pre-LHC posterior distributions (dashed lines) and the surviving parameter regions (solid-blue lines) after imposing both $m_{h}=$ $122.0-128.0 \mathrm{GeV}$ and $1.67-\sigma R_{Z Z ; \gamma \gamma}$. All the masses are in TeV units. The vertical axes represent the relative probability weights of the model points. 

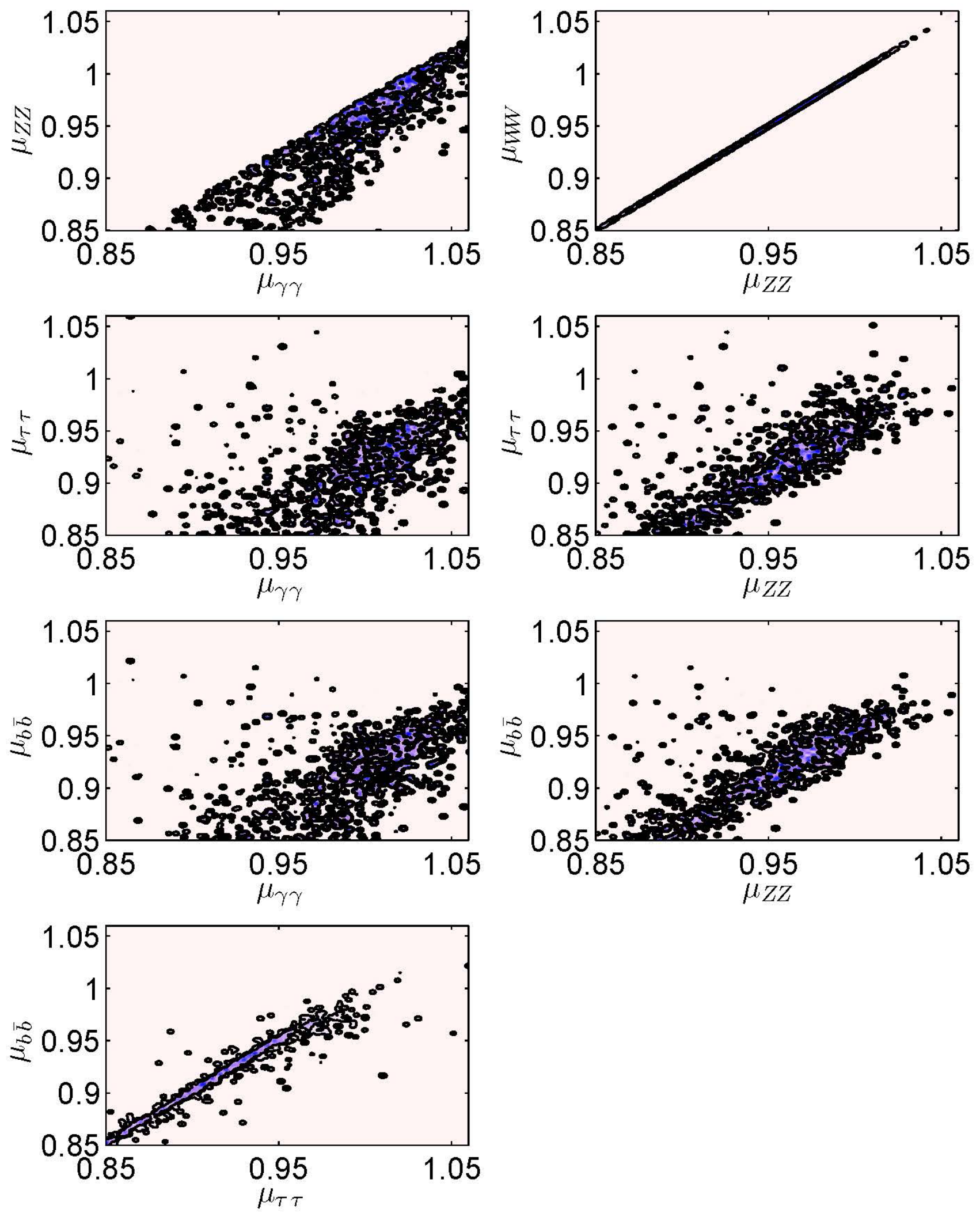

Figure 3. Marginalised posterior distributions for the signal strengths at $8 \mathrm{TeV}$ LHC for the pMSSM points that survive the $m_{h}=122-128 \mathrm{GeV}$ and $1.67-\sigma R_{Z Z ; \gamma \gamma}$ constraints. The colour spread from light pink to blue represents relative probability spread from zero to unity. The inner and outer contours represent $68 \%$ and $95 \%$ probability regions respectively. The distributions for the flat prior sample is approximately the same but with narrower peaks around unity. 
the posterior distributions for a pre-LHC Bayesian fit to the pMSSM, sorting for the impact of the discovery data on the sparticle mass predictions. The data provides mainly two classes of information; one about the mass and the other about the signal strength of the new state.

The pre-LHC pMSSM fits predict an approximately prior-independent Higgs boson mass to be between 117 GeV and $129 \mathrm{GeV}$ at $95 \%$ Bayesian confidence level and, hence, are in agreement with the recent discovery of a new state around $126 \mathrm{GeV}$. The pMSSM's signal strengths are narrowly distributed around unity, as shown in Fig. 1 suggesting compatibility with the SM expectations. These are in agreement with the experimentally determined values, to within $2 \sigma$ (or $95 \%$ C.L.) for the diphoton channel. This also holds for the aforementioned discriminant, simply as a consequence of the as yet large error bars associated with it. In other words, at this level, the effect of the signal strengths is not yet conclusive, and better precisions in the results is necessary for making distinctive conclusions.

On the other hand, if we demand that the MSSM expectation of the discriminant agree with the data to within $1 \sigma$, almost none, viz. $0.0 \%$ (3 model points) of the flat prior and some $0.3 \%$ (125 model points) of the log prior posterior samples survive. Indeed, even at $90 \%$ C.L., the constraints are quite strong. The surviving regions of the parameter space typically are associated with lighter stau states and lighter 1st/2nd-generation squarks, whereas the stops, the charginos and the (non-LSP) neutralinos tend to be heavy. The prediction for light squarks is apparently in tension with limits from SUSY searches. However, it should be realized that the purpose here is to look for the directions in sparticle mass space to which the Higgs data pull, and the tension is not an overwhelming one. As for the stau states, the current direct LHC limits from either (Drell-Yan like) pair-production or staus from weak gaugino decays are weak. Future limits on the stau mass would be important constraints for the $1 \sigma$ $R_{Z Z ; \gamma \gamma}$ surviving points.

In conclusion, assuming an R-parity conserving MSSM, the results of our analyses imply that if the diphoton signal strength anomaly persists even after the accumulation of more LHC data, then it is very likely that there exist light sparticles which have to-date escaped detection. This could be due to the low magnitude of missing energy in the case of light (compared to the experimental missing transverse energy cuts) neutralinos and/or due to the quasi-degenerate nature of the spectrum which leads to soft jets [29,30] that would be buried under QCD background $^{8}$. Given the relatively low statistics for the surviving points, it is now time for updating statistically robust trends for MSSM physics by performing new pMSSM explorations and global fits to the post-discovery LHC data. Before this, short-range outlook indicates that performing a similar study, but for the impact of the LHC SUSY limits on the pMSSM posterior distributions is well within reach [33]. The same is the case for checking the impact on the freedom of choice for considering the LSP as being either the only or a partial source for CDM relic density within the pMSSM framework [34]. Other interesting directions include searching for plausible effects of non-diagonal flavour on the pMSSM predictions and for computing the impacts of the discovery data and SUSY limits on a phenomenological next-to-MSSM, the pNMSSM.

\section{Acknowledgments}

We thank F. Quevedo for useful comments and discussions. DC thanks the Abdus Salam ICTP for hospitality during the period when this project was conceptualised and started.

\section{REFERENCES}

[1] G. Aad et al. [ATLAS Collaboration], "Observation of a new particle in the search for the Standard Model Higgs boson with the ATLAS detector at the LHC," Phys. Lett. B 716 (2012) 1 [arXiv:1207.7214 [hep-ex]].

[2] S. Chatrchyan et al. [CMS Collaboration], "Observation of a new boson at a mass of $125 \mathrm{GeV}$ with the CMS experiment at the LHC," Phys. Lett. B $\mathbf{7 1 6}$ (2012) 30 [arXiv:1207.7235 [hep-ex]].

[3] I. A. Oksuzian [CDF and D0 Collaborations], "Searches for the Higgs boson at the Tevatron," arXiv:1209.1586 [hep-ex].

[4] J. Baglio, A. Djouadi and R. M. Godbole, "The apparent excess in the Higgs to di-photon rate at the LHC: New Physics or QCD uncertainties?," Phys. Lett. B 716 (2012) 203 [arXiv:1207.1451 [hep-ph]].

[5] H.E. Haber, Nucl. Phys. B. (Proc. Suppl.) 62A-C, 469 (1998).

[6] A. Djouadi et al. [MSSM Working Group Collaboration], "The Minimal supersymmetric standard model: Group summary report," hep-ph/9901246.

[7] S. S. AbdusSalam, "The Full 24-Parameter MSSM Exploration," AIP Conf. Proc. 1078 (2009) 297 [arXiv:0809.0284 [hep-ph]].

[8] S. S. AbdusSalam, B. C. Allanach, F. Quevedo, F. Feroz and M. Hobson, "Fitting the Phenomenological MSSM," Phys. Rev. D 81 (2010) 095012 [arXiv:0904.2548 [hep-ph]].

[9] J. A. Conley, J. S. Gainer, J. L. Hewett, M. P. Le and T. G. Rizzo, "Supersymmetry Without Prejudice at the 7 TeV LHC," [arXiv:1103.1697 [hep-ph]].

\footnotetext{
${ }^{8}$ It is not obvious if the compressed SUSY scenario [31] considered within a modified-CMSSM/mSUGRA benchmarking models can survive with Higgs boson mass around $126 \mathrm{GeV}[32]$.
} 
[10] B. C. Allanach, A. J. Barr, A. Dafinca and C. Gwenlan, "Discovery reach for generic supersymmetry at the LHC: MT2 versus missing transverse momentum selections for pMSSM searches," JHEP 1107 (2011) 104 [arXiv:1105.1024 [hep-ph]].

[11] S. Sekmen, S. Kraml, J. Lykken, F. Moortgat, S. Padhi, L. Pape, M. Pierini and H. B. Prosper et al., "Interpreting LHC SUSY searches in the phenomenological MSSM," JHEP 1202 (2012) 075 [arXiv:1109.5119 [hep-ph]].

[12] A. Arbey, M. Battaglia and F. Mahmoudi, "Implications of LHC Searches on SUSY Particle Spectra: The pMSSM Parameter Space with Neutralino Dark Matter," Eur. Phys. J. C 72 (2012) 1847 [arXiv:1110.3726 [hep-ph]].

[13] D. Albornoz Vasquez, G. Belanger, R. M. Godbole and A. Pukhov, "The Higgs boson in the MSSM in light of the LHC," Phys. Rev. D 85 (2012) 115013 [arXiv:1112.2200 [hep-ph]].

[14] A. Djouadi, V. Driesen, W. Hollik and J. I. Illana, "The Coupling of the lightest SUSY Higgs boson to two photons in the decoupling regime," Eur. Phys. J. C 1 (1998) 149 [hep-ph/9612362].

[15] A. Djouadi, "Squark effects on Higgs boson production and decay at the LHC," Phys. Lett. B 435 (1998) 101 [hep$\mathrm{ph} / 9806315]$.

[16] A. Arbey, M. Battaglia, A. Djouadi and F. Mahmoudi, "The Higgs sector of the phenomenological MSSM in the light of the Higgs boson discovery," arXiv:1207.1348 [hep-ph].

[17] M. Carena, S. Gori, N. R. Shah, C. E. M. Wagner and L. -T. Wang, "Light Stau Phenomenology and the Higgs gamma gamma Rate," JHEP 1207 (2012) 175 [arXiv:1205.5842 [hep-ph]]. Cao:2012fz,

[18] J. -J. Cao, Z. -X. Heng, J. M. Yang, Y. -M. Zhang and J. -Y. Zhu, JHEP 1203 (2012) 086 [arXiv:1202.5821 [hep-ph]].

[19] J. Cao, Z. Heng, J. M. Yang and J. Zhu, JHEP 1210 (2012) 079 [arXiv:1207.3698 [hep-ph]].

[20] O. Eberhardt, G. Herbert, H. Lacker, A. Lenz, A. Menzel, U. Nierste and M. Wiebusch, "Impact of a Higgs boson at a mass of $126 \mathrm{GeV}$ on the standard model with three and four fermion generations," arXiv:1209.1101 [hep-ph].

[21] M. Botje, J. Butterworth, A. Cooper-Sarkar, A. de Roeck, J. Feltesse, S. Forte, A. Glazov and J. Huston et al., "The PDF4LHC Working Group Interim Recommendations," arXiv:1101.0538 [hep-ph].

[22] S. Dittmaier et al. [LHC Higgs Cross Section Working Group Collaboration], "Handbook of LHC Higgs Cross Sections: 1. Inclusive Observables," arXiv:1101.0593 [hep-ph].

[23] A. Arvanitaki and G. Villadoro, "A Non Standard Model Higgs at the LHC as a Sign of Naturalness," JHEP 1202 (2012) 144 [arXiv:1112.4835 [hep-ph]].

[24] J. Guasch, W. Hollik and S. Penaranda, "Distinguishing Higgs models in H - i b anti-b / H - i tau+ tau-," Phys. Lett. B 515 (2001) 367 [hep-ph/0106027].

[25] K. A. Assamagan, J. Guasch, S. Moretti and S. Penaranda, "Determining the ratio of the H+ - i tau nu to H+ - i t anti-bb-bar decay rates for large tan beta at the large hadron collider," hep-ph/0402212.

[26] K. A. Assamagan, J. Guasch, S. Moretti and S. Penaranda, "Distinguishing Higgs models in H+ - i tau+ nu / t anti-b at large tan beta," Czech. J. Phys. 55 (2005) B787 [hep-ph/0409189].

[27] S. Heinemeyer, W. Hollik and G. Weiglein, "FeynHiggs: A Program for the calculation of the masses of the neutral CP even Higgs bosons in the MSSM," Comput. Phys. Commun. 124 (2000) 76 [hep-ph/9812320]; R. V. Harlander and W. B. Kilgore, Phys. Rev. D 68 (2003) 013001 [hep-ph/0304035]; S. Catani, D. de Florian and M. Grazzini, JHEP 0105 (2001) 025; R. V. Harlander and W. B. Kilgore, Phys. Rev. Lett. 88 (2002) 201801; C. Anastasiou and K. Melnikov, Nucl. Phys. B 646 (2002) 220; V. Ravindran, J. Smith and W. L. van Neerven, Nucl. Phys. B 665 (2003) 325; C. Anastasiou, R. Boughezal, F. Petriello, JHEP 0904 (2009) 003.

[28] G. Degrassi, S. Heinemeyer, W. Hollik, P. Slavich and G. Weiglein, "Towards high precision predictions for the MSSM Higgs sector," Eur. Phys. J. C 28 (2003) 133 [hep-ph/0212020].

[29] S. S. AbdusSalam, "Can the LHC rule out the MSSM?," Phys. Lett. B 705 (2011) 331 [arXiv:1106.2317 [hep-ph]].

[30] S. S. AbdusSalam, B. C. Allanach, H. K. Dreiner, J. Ellis, U. Ellwanger, J. Gunion, S. Heinemeyer and M. Kraemer et al., "Benchmark Models, Planes, Lines and Points for Future SUSY Searches at the LHC," Eur. Phys. J. C 71 (2011) 1835 [arXiv:1109.3859 [hep-ph]].

[31] T. J. LeCompte and S. P. Martin, "Large Hadron Collider reach for supersymmetric models with compressed mass spectra," Phys. Rev. D 84 (2011) 015004 [arXiv:1105.4304 [hep-ph]].

[32] Editors: R. Cavanaugh, J . L. Hewett, S. Kraml, M. L. Mangano, G. Polesello Contributing authors: S. S. AbdusSalam, B. C. Allanach, A. Arbey, D. S. M. Alves, H. Baer, M. Battaglia, G. Belanger and F. Brümmer et al., "Implications of LHC results for TeV-scale physics: new physics with missing energy signatures," Submitted to the Open Symposium of the European Strategy Preparatory Group, 10-12 September 2012 Krakow, Poland.

[33] S. S. AbdusSalam, "LHC-7 supersymmetry search interpretation within the pMSSM," arXiv:1211.0999 [hep-ph].

[34] S. S. AbdusSalam and F. Quevedo, "Cold Dark Matter Hypotheses in the MSSM," Phys. Lett. B 700 (2011) 343 [arXiv:1009.4308 [hep-ph]]. 